\title{
Determinação da Biodisponibilidade da Lisina Sulfato e Lisina HCl com Frangos de Corte ${ }^{1}$
}

\section{Rafael Neme ${ }^{2}$, Luiz Fernando Teixeira Albino ${ }^{3}$, Horacio Santiago Rostagno ${ }^{3}$, Ramalho José Barbosa Rodrigueiro4, Rodrigo Santana Toledo ${ }^{4}$}

\begin{abstract}
RESUMO - Com o objetivo de determinar a biodisponibilidade de duas fontes de lisina (lisina HCl e lisina sulfato), por intermédio de um ensaio de crescimento, foram alojados em um galpão de alvenaria com 56 boxes 840 pintos de corte machos com um dia de idade. Duas dietas basais foram formuladas para atender as exigências nutricionais das aves nas fases inicial e crescimento, deficientes apenas em lisina e suplementadas em 0,$08 ; 0,16$; e 0,24\% pelas duas fontes de lisina. As variáveis avaliadas foram: ganho de peso, consumo de ração, conversão alimentar, rendimento de carcaça, rendimento de perna, rendimento de peito, rendimento de filé e porcentagem de gordura abdominal. Com os dados obtidos foram estimadas equações de regressão linear múltipla e, usando os coeficientes de regressão destas, foi determinada a biodisponibilidade da lisina sulfato em relação a lisina $\mathrm{HCl}$, padronizada como $100 \%$ disponível. As equações obtidas que melhor estimaram a biodisponibilidade das lisinas foram $\mathrm{Y}=544,72+439,62 \mathrm{X}_{1}+475,84 \mathrm{X}_{2}, \mathrm{R}^{2}=0,90$, para ganho de peso de 01 a 21 dias de idade, $\mathrm{Y}=1824,63+1469,18 \mathrm{X}_{1}+1381,33 \mathrm{X}_{2}, \mathrm{R}^{2}=0,85$, para ganho de peso de 01 a 42 dias de idade, $\mathrm{Y}=1,9623-0,9043 \mathrm{X}_{1}-1,0235 \mathrm{X}_{2}, \mathrm{R}^{2}=0,83$, para conversão alimentar de 01 a 21 dias de idade, $\mathrm{Y}=0,3766+0,5320 \mathrm{X}_{1}+0,4986 \mathrm{X}_{2}$, $\mathrm{R}^{2}=0,88$, para peso de peito aos 42 dias de idade e $\mathrm{Y}=0,2565+0,4685 \mathrm{X}_{1}+0,4300 \mathrm{X}_{2}, \mathrm{R}^{2}=0,92$, para peso de filé de peito aos 42 dias de idade das aves. A biodisponibilidade média encontrada para a Lisina Sulfato foi de 100,19\%, mostrando não haver diferença significativa na biodisponibilidade das lisinas testadas.
\end{abstract}

Palavras-chave: biodisponibilidade, ensaio de crescimento, frango de corte, lisina

\section{Bioavailability Determination of Lysine Sulfate and Lysine HCl with Broiler Chickens}

ABSTRACT - With the objective of determine the bioavailability of two lysine sources (lysine $\mathrm{HCl}$ and lysine sulfate), by a growth trial, 840 one day old male broiler chicks were placed in 56 boxes. Two basal diets were formulated to supply the birds nutritional requirements, one for the starting and the other for the growing period, respectively, deficient only in lysine, which were supplemented $0,08,0,16$ and $0,24 \%$ lysine of both lysine sources. Weight gain, feed intake, feed conversion, carcass yield, legs quarter yield, breast yield, breast meat yield and abdominal fat content were evaluated. Multiple linear regression and the slope ratio technique was used to estimate equations. The bioavailabity of lysine sulfate was obtained as a percentage of lysine $\mathrm{HCl}$ considered as $100 \%$ available. The equations that best estimated the bioavailability of the lysine sulfate $\left(\mathrm{X}_{2}\right)$ related to lysine $\mathrm{HCl}\left(\mathrm{X}_{1}\right)$ were: $\mathrm{Y}=544,72+439,62 \mathrm{X}_{1}+$ $475,84 \mathrm{X}_{2}, \mathrm{R} 2=0,90$, for weight gain ( 1 to 21 days of age); $\mathrm{Y}=1824,63+1469,18 \mathrm{X}_{1}+1381,33 \mathrm{X}_{2}$, $\mathrm{R}^{2}=0,85$, for weight gain from 01 to 42 days of age; $11 \mathrm{Y}=1,9623-0,9043 \mathrm{X}_{1}-1,0235 \mathrm{X}_{2}, \mathrm{R}^{2}=0,83$, for feed conversion ( 1 to 21 days of age); $\mathrm{Y}=0,3766+0,5320$ $\mathrm{X}_{1}+0,4986 \mathrm{X}_{2}, \mathrm{R}^{2}=0,88$, for breast yield (42 days of age) and $\mathrm{Y}=0,2565+0,4685 \mathrm{X}_{1}+0,4300 \mathrm{X}_{2}, \mathrm{R}^{2}=0,92$ for breast meat yield with 42 days of age. The average bioavailability of lysine sulfate was 100,19\%, showing that there was no difference ( $\mathrm{p}>0.05$ ), in the bioavailability of the two lysine sources studied.

Key Words: bioavailability, broiler, grow trial, lysine

\section{Introdução}

Os frangos de corte comerciais são aves que apresentam crescimento rápido e de maior eficiência já produzidos pelos esforços combinados entre o homem e a natureza. No entanto, para obtermos uma máxima resposta fenotípica dessas aves, em função do enorme potencial genético atingido, devemos proporcionar a esses animais o máximo em qualidade ambiental e nutricional. A avicultura no mundo todo tem um objetivo comum que é a obtenção de produtos avícolas de alta qualidade, juntamente com menores custos de produção. Uma vez que, do custo total de produção na avicultura cerca de $70 \%$ é devido a alimentação, tendo os alimentos protéicos grande contribuição neste custo total, fica evidente a grande importância da estratégia nutricional a ser utilizada dentro de uma empresa. Uma das alternativas utiliza-

\footnotetext{
${ }_{1}^{1}$ Parte da tese de Mestrado do primeiro autor.

2 Estudante de Doutorado FACV - UNESP/Jaboticabal.

3 Professor Titular DZO/UFV. E.mail: lalbino@ufv.br; rostagno@ufv.br
}

4 Estudante de Pós-graduação DZO/UFV. 
das com o intuito de redução dos custos é a diminuição do nível protéico das rações adicionando aminoácidos sintéticos em níveis que proporcione às aves índices de desempenho semelhantes ou até melhores.

Entre os aminoácidos de maior importância na cadeia produtiva avícola e suinícola PARSONS e BAKER (1994) destacaram metionina + cistina, lisina, treonina, triptofano e arginina.

Segundo ALBINO (1991), para se obter uma ração econômica e adequada nutricionalmente, devem-se considerar as necessidades das aves em aminoácidos disponíveis bem como os aminoácidos disponíveis nos alimentos, principalmente quando alimentos alternativos são utilizados em substituição ao milho e ao farelo de soja. Em rações à base de farelo de soja e milho, alimentos mais utilizados hoje em dia na avicultura, tem-se como aminoácidos limitantes a metionina + cistina e lisina, para aves e suínos respectivamente.

Os aminoácidos limitantes são aqueles que estão presentes nas dietas em concentrações menores que as exigidas para o máximo de síntese protéica, EASTER (1985).

Segundo WALDROUP et al. (1976) o desequilíbrio nutricional de uma ração pode ocasionar prejuízos ao desempenho dos animais. O desbalanço de aminoácidos, resulta em vários problemas sendo um deles a relação existente entre aminoácidos antagônicos, como lisina e arginina. $\mathrm{O}$ antagonismo entre aminoácidos é uma interação que envolve os aminoácidos estruturalmente semelhantes, sendo que o excesso de um eleva a exigência do outro, AUSTIC (1985).

Embora a lisina seja o segundo aminoácido limitante para frangos de corte, ela é usada como aminoácido referência na formulação de rações com base na proteína ideal, pois é utilizada exclusivamente para acréscimo de proteína corporal, em contraste com a metionina e a cistina, que são utilizadas por diferentes caminhos metabólicos, como manutenção e plumagem (PACK, 1995).

Segundo BAKER et al. (1994), a lisina é utilizada como aminoácido referência por três razões principais; 1) sua análise nos alimentos é relativamente simples, diferente de triptofano e dos aminoácidos sulfurados; 2) há grande quantidade de dados existentes sobre a digestibilidade da lisina em aves; 3 ) diferente de vários aminoácidos (metionina, cistina e triptofano), a absorção de lisina é utilizada somente para acréscimo de proteína corporal.
Com as expressivas pesquisas sobre exigências nutricionais de aminoácidos para frango de corte, principalmente metionina e lisina, juntamente com o aparecimento de aminoácidos sintéticos de diferentes formas como, DL-Metionina, L-Metionina, Metionina Hidróxi Análoga - Ácido Livre, L-Lisina $\mathrm{HCl}$, L-Lisina Líquida e Lisina Sulfato, tornou-se possível a formulação de rações que atendam com maior precisão as exigências das aves e com menores custos.

Dessa forma, o objetivo deste trabalho foi determinar a biodisponibilidade de duas fontes de lisina disponíveis no mercado (Lisina $\mathrm{HCl}$ e Lisina Sulfato).

\section{Material e Métodos}

Foram utilizados 840 pintos de corte machos, da marca comercial Cobb, com um dia de idade e alojados em um galpão de alvenaria subdividido em 56 boxes de 1,50 x 1,70 metros. Em cada boxe foram colocadas 20 aves, apresentando peso médio/boxe/ ave de 47 gramas, e criadas até 42 dias de idade. As rações experimentais e a água foram fornecidas à vontade, sendo o manejo utilizado durante todo o período experimental semelhante às criações comerciais. As temperaturas máximas e mínimas foram anotadas diariamente.

Aos 21 e 42 dias de idade, as aves e a sobra de ração de cada unidade experimental foram pesadas. Aos 42 dias, após pesagem das unidades experimentais as aves foram pesadas individualmente para a escolha de quatro aves por boxe que representassem o peso médio desta unidade experimental. Estas foram devidamente identificadas $\left(\mathrm{N}^{\mathrm{O}}\right.$ do boxe e $\mathrm{N}^{\mathrm{O}} \mathrm{da}$ ave dentro do boxe) e abatidas para avaliação de rendimento de carcaça, rendimento de perna (coxa e sobrecoxa), rendimento de peito, rendimento de filé de peito e quantidade de gordura abdominal.

Foi utilizado delineamento experimental inteiramente casualizado, com sete tratamentos e seis repetições de 20 aves por unidade experimental. Os tratamentos consistiram de três níveis de lisina Sulfato, três níveis de Lisina $\mathrm{HCl}$ e uma ração basal deficiente em lisina.

Tratamento 1 - Ração Basal

Tratamento 2 - Ração Basal $+0,08 \%$ de lisina (L-lisina $\mathrm{HCl}=0,101 \%$ )

Tratamento 3 - Ração Basal $+0,16 \%$ de lisina (L-lisina $\mathrm{HCl}=0,203 \%)$

Tratamento 4 - Ração Basal + 0,24\% de lisina 
( $\mathrm{L}$ - lisina $\mathrm{HCl}=0,304 \%)$

Tratamento 5 - Ração Basal + 0,08\% de lisina $($ Lisina Sulfato $=0,171 \%)$

Tratamento 6 - Ração Basal $+0,16 \%$ de lisina (Lisina Sulfato $=0,342 \%$ )

Tratamento 7 - Ração Basal + 0,24\% de lisina (Lisina Sulfato $=0,513 \%)$

Nas duas fases de criação (01 a 21 e 22 a 42 dias), as rações basais foram calculadas para atender as exigências nutricionais das aves de acordo com ROSTAGNO et al. (1996), para todos os nutrientes, exceto lisina, sendo então suplementadas com diferentes níveis deste aminoácido. A técnica de diluição de rações proposta por GOUS e MORRIS (1985), foi utilizada para a obtenção dos três níveis de cada fonte de lisina.

As rações experimentais nas duas fases de criação, foram isocalóricas e isoprotéicas, variando apenas os níveis e a fonte de lisina de acordo com a Tabela 1.

As análises estatísticas das características estudadas foram realizadas de acordo com o programa SAEG (Sistemas de Análises Estatísticas e Genéticas) - UFV, versão 8.0 (2000).

Foi feita análise de variância considerando os sete tratamentos. Outra análise de variância foi realizada, considerando apenas a dieta basal e os níveis

Tabela 1 - Composição percentual das rações basais

Table 1 - Percentage composition of basal diets

\begin{tabular}{|c|c|c|}
\hline $\begin{array}{l}\text { Ingredientes } \\
\text { Ingredients }\end{array}$ & $\begin{array}{l}\text { Inicial } \\
\text { Starter }\end{array}$ & $\begin{array}{l}\text { Crescimento } \\
\text { Growing }\end{array}$ \\
\hline Milho (Corn) & 48,125 & 51,95 \\
\hline Farelo de soja (Soybean meal) $45 \%$ & 21,800 & 18,19 \\
\hline Farinha carne e ossos $40 \%$ (Meat and bone meal) & 3,000 & 3,500 \\
\hline Sorgo baixo tanino (Sorghum low tanin) & 15,000 & 15,00 \\
\hline Glúten de milho (Corn gluten feed) & 8,000 & 6,500 \\
\hline Amido (Corn starch) & 0,670 & 0,650 \\
\hline Calcário (Limestone) & 0,600 & 0,500 \\
\hline Fosfato bicálcico (Dicalcium phosphate) & 0,800 & 0,600 \\
\hline Óleo vegetal (Soybean oil) & 1,060 & 2,200 \\
\hline Sal comum (Salt) & 0,350 & 0,350 \\
\hline Mistura mineral $^{1}$ (Mineral premix) & 0,050 & 0,050 \\
\hline Mistura vitamínica ${ }^{2}$ (Vitamin premix) & 0,100 & 0,100 \\
\hline Stafac $^{3}$ & 0,075 & 0,050 \\
\hline Anticoccidiano ${ }^{4}$ (Coccidiostatic) & 0,060 & 0,060 \\
\hline L-Treonina 99\% (L-threonine 99\%) & 0,025 & 0,020 \\
\hline DL-Metionina 98\% (DL-Methionine 98\%) & 0,180 & 0,190 \\
\hline CL-Colina, $60 \%$ (Choline chlorine) & 0,100 & 0,060 \\
\hline L-Triptofano (L-Tryptofan) & - & 0,020 \\
\hline BHT & 0,010 & 0,010 \\
\hline Total & 100,00 & 100,00 \\
\hline \multicolumn{3}{|l|}{ Valores calculados (Calculated composition) } \\
\hline Proteína bruta, \% (Crude protein) & 21,775 & 19,731 \\
\hline $\mathrm{EM}(M E), \mathrm{kcal} / \mathrm{kg}$ & 3,101 & 3,205 \\
\hline $\mathrm{Ca}(\%)$ & 0,978 & 0,956 \\
\hline P disponível, \% (Available $P$ ) & 0,455 & 0,447 \\
\hline Metionina, \% (Methionine) & 0,573 & 0,524 \\
\hline Metionina + Cistina,$\%$ (Methionine + Cystine) & 0,931 & 0,869 \\
\hline Met + Cis digestível, \% (Met + digestible Cys $)$ & 0,847 & 0,791 \\
\hline Lisina total, \% (Total lysine) & 0,911 & 0,809 \\
\hline Lisina digestível, \% (Digestible lysine) & 0,806 & 0,712 \\
\hline Treonina, \% (Threonine) & 0,829 & 0,747 \\
\hline Treonina digestível, \% (Digestible threonine) & 0,663 & 0,599 \\
\hline Triptofano, \% (Tryptophan) & 0,235 & 0,210 \\
\hline
\end{tabular}

${ }^{1}$ Níveis de garantia por quilo de produto: $\mathrm{Fe}, 80 \mathrm{~g}, \mathrm{Cu}, 10 \mathrm{~g}, \mathrm{Co}, 2 \mathrm{~g}, \mathrm{Mn}, 60 \mathrm{~g}, \mathrm{Zn}, 50 \mathrm{~g}, \mathrm{l}, 2 \mathrm{~g}$, qsq - $500 \mathrm{~g}$.

2 Níveis de garantia por quilo de produto, vit. $A-15.000 .000 \mathrm{UI}$, vit. $D_{3}-1.500 .000$, Ul, vit. $B_{1}-2 \mathrm{~g}$, vit. $B_{2}-4 \mathrm{~g}$, vit. $B_{6}-3 \mathrm{~g}$, vit. $\mathrm{B}_{12}-0,015 \mathrm{~g}$, ácido nicotínico (nicotinic acid) $-10 \mathrm{~g}$, vit. $\mathrm{K}_{3}-3 \mathrm{~g}$, ácido fólico (folic acid) $-1,0 \mathrm{~g}$, bacitracina de, selênio $-250 \mathrm{mg}$, BHT - 10, qsq $1.000 \mathrm{~g}$

3 Stafac $2 \%$, virginiamicina - $15 \mathrm{ppm}(750 \mathrm{~g} / \mathrm{t})$

4 Coban 200 - monensina sódica $120 \mathrm{ppm}(600 \mathrm{~g} / \mathrm{t})$.

${ }^{5}$ Análises realizadas no laboratório da Degussa - Hulls A G, Feed aditive division. 
de suplementação de uma mesma fonte de lisina, com a finalidade de verificar a ocorrência de efeito linear nos níveis de suplementação dentro de cada fonte (Lisina $\mathrm{HCl}$ e Lisina Sulfato).

Para determinação da biodisponibilidade da Lisina Sulfato em relação a Lisina $\mathrm{HCl}$ (considerada como $100 \%$ disponível), utilizou-se a relação dos coeficientes de regressão (SASSE e BAKER, 1973), adotando o seguinte modelo.

Regressão Linear Múltipla: $y=a+b_{1} X_{1}+b_{2} X_{2}$, em que: $\mathrm{y}=$ valores observados das características ganho de peso, consumo de ração e conversão alimentar; $a=$ intercepto; $b_{1}$ e $b_{2}=$ coeficientes de regressão para lisina $\mathrm{HCl}$ e Lisina Sulfato, respectivamente; e $\mathrm{X}_{1}$ e $\mathrm{X}_{2}=$ nível suplementar de lisina $\mathrm{HCl}$ e Lisina Sulfato, respectivamente.

As características estudadas foram: consumo de ração $(\mathrm{kg})$, ganho de peso $(\mathrm{kg})$, conversão alimentar, nos dois períodos de criação das aves e no período total (01 a 42 dias). Aos 42 dias foram avaliados o rendimento de carcaça $(\%)$, rendimento de perna $(\%)$, (coxa e sobrecoxa), rendimento de peito (\%), rendimento de filé de peito (\%), (peito sem pele e sem osso), e rendimento de gordura abdominal (\%).

$\mathrm{O}$ rendimento de carcaça (\%) foi obtido pela relação entre o peso da carcaça eviscerada (sem pés, cabeça e pescoço), e o peso vivo. Os rendimentos de perna, peito, filé de peito e gordura abdominal foram obtidos em função da carcaça eviscerada.

\section{Resultados e Discussão}

Ganho de peso, consumo de ração e conversão alimentar

As médias dos resultados de ganho de peso, consumo de ração e conversão alimentar referentes as dietas; basal, suplementada com Lisina $\mathrm{HCl}$ e suplementada com Lisina Sulfato para os períodos de 1 a 21, 22 a 42 e 1 a 42 dias de idade das aves encontram-se nas Tabelas 2, 3 e 4, respectivamente.

Como mostrado na Tabela 2 foi detectada diferença $(\mathrm{P}<0,05)$ para conversão alimentar das aves criadas de 01 a 21 dias de idade em relação às duas fontes de lisina. A Lisina Sulfato proporcionou uma eficiência na utilização do alimento $3,42 \%$ superior à Lisina $\mathrm{HCl}$. No entanto, considerando os níveis analisados de lisina nas diferentes rações, pode-se notar 3,20\% de Lisina Sulfato $(1,03 \%)$ a mais que a Lisina $\mathrm{HCl}(0,997 \%)$, abrindo margens para um questionamento desta maior eficiência alimentar encontrada.

Tabela 2 - Efeito da fonte de lisina, em diferentes níveis, no desempenho de frangos de corte de 1 a 21 dias Table 2 - Effect of lysine source, different levels, on broiler performance from 1 to 21 days

\begin{tabular}{|c|c|c|c|c|}
\hline $\begin{array}{l}\text { Tratamentos } \\
\text { Treatments }\end{array}$ & $\begin{array}{c}\text { Lisina }(\%) \\
\text { Lysine } \\
\text { Calculado } \backslash \text { Analisado } \\
\text { Calculated } \backslash \text { Analyzed }\end{array}$ & $\begin{array}{c}\text { Ganho de peso }(\mathrm{g}) \\
\text { Body weight gain }\end{array}$ & $\begin{array}{c}\text { Consumo de ração }(\mathrm{g}) \\
\text { Feed intake }\end{array}$ & $\begin{array}{l}\text { Conv. alimentar } \\
\text { Feed:gain ratio }\end{array}$ \\
\hline $\begin{array}{l}\text { Dieta basal } \\
\text { Basal diet }\end{array}$ & $0,911 \backslash 0,850$ & $526^{\mathrm{b}}$ & $1059^{b}$ & $2,018^{\mathrm{a}}$ \\
\hline $\begin{array}{l}\text { Lisina } \mathrm{HCl} 0,08 \\
\text { Lysine }\end{array}$ & $0,991 \backslash 0,930$ & 595 & 1111 & 1,866 \\
\hline $\begin{array}{l}\text { Lisina } \mathrm{HCl} 0,16 \\
\text { Lysine }\end{array}$ & $1,071 \backslash 1,000$ & 619 & 1121 & 1,810 \\
\hline $\begin{array}{l}\text { Lisina } \mathrm{HCl} 0,24 \\
\text { Lysine }\end{array}$ & $1,151 \backslash 1,060$ & 640 & 1125 & 1,757 \\
\hline $\begin{array}{l}\text { Média } \\
\text { Mean }\end{array}$ & $1,071 \backslash 0,997$ & $\begin{array}{l}618^{\mathrm{a}} \\
\mathrm{L} *\end{array}$ & $\begin{array}{l}1119^{\mathrm{a}} \\
\mathrm{NS}\end{array}$ & $\begin{array}{c}1,813^{\mathrm{b}} \\
\mathrm{L} * *\end{array}$ \\
\hline $\begin{array}{l}\text { Lisina sulfato } 0,08 \\
\text { Lysine sulfate }\end{array}$ & $0,911 \backslash 0,970$ & 604 & 1088 & 1,800 \\
\hline $\begin{array}{l}\text { Lisina sulfato } 0,16 \\
\text { Lysine sulfate }\end{array}$ & $1,071 \backslash 1,010$ & 651 & 1128 & 1,733 \\
\hline $\begin{array}{l}\text { Lisina sulfato } 0,24 \\
\text { Lysine sulfate }\end{array}$ & $1,151 \backslash 1,110$ & 661 & 1136 & 1,719 \\
\hline $\begin{array}{l}\text { Média } \\
\text { Mean } \\
\text { CV }(\%)\end{array}$ & $1,071 \backslash 1,030$ & $\begin{array}{l}639^{\mathrm{a}} \\
\mathrm{L} * * \\
5,547\end{array}$ & $\begin{array}{c}117^{\mathrm{a}} \\
\mathrm{NS} \\
4,074\end{array}$ & $\begin{array}{l}1,753^{\mathrm{c}} \\
\mathrm{L} * * * \\
3,801\end{array}$ \\
\hline
\end{tabular}

NS Não significativo (Not significant). ${ }^{*}$ Efeito Linear (Linear effect) $(P<0,05) .{ }^{* *}$ Efeito Linear (Linear effect) $(P<0,01) .{ }^{* * *}$ Efeito Linear $($ Linear effect) $(\mathrm{P}<0,001)$.

Médias nas mesma coluna seguidas de letras diferentes, diferem $(P<0,05)$, pelo teste de Newman-Keuls.

Means in the same column followed by different letters are different by Newman Keuls test. 
Como observado nas Tabelas 2, 3 e 4, a adição das diferentes fontes de lisina (lisina $\mathrm{HCl}$ e lisina sulfato) proporcionou melhora significativa $(\mathrm{P}<0,05)$, para as variáveis estudadas em relação a ração basal, não ocorrendo diferença entre as diferentes fontes deste aminoácido.

A diferença observada nos parâmetros de desempenho entre a média da ração basal e as médias das rações suplementadas com lisina eram esperadas uma vez que a ração basal foi deficiente neste aminoácido. EMMERT et al. (1999) confrontando duas fontes de lisina com uma ração deficiente neste aminoácido encontraram resultados semelhantes.

Quando as aves receberam uma ração deficiente em um aminoácido, constatou-se aumento no consumo de ração das aves e, conseqüentemente, do aminoácido deficitário, para suprimento da demanda de mantença e produção (GOUS e MORRIS, 1985). Este maior consumo de ração foi observado por BARBOSA (1998), ao fornecer dietas com níveis deficientes em metionina + cistina. No entanto, os resultados obtidos no presente trabalho mostram que o consumo de ração das aves submetidas à ração basal, deficiente em lisina, foi estatisticamente menor $(\mathrm{P}<0,05)$ que o das dietas suplementadas com lisina de ambas as fontes, sugerindo que, quando deficientes nas rações, a lisina proporciona um comportamento diferenciado dos demais aminoácidos.

Foi verificado também um maior consumo de ração por quilo de ganho das aves que receberam a ração basal, mostrando que a deficiência de lisina afeta não só o consumo de ração mas também a eficiência de utilização do alimento. A melhora na eficiência de utilização do alimento, ou seja, menor conversão alimentar, foi observada por EMMERT et al. (1999) e GOUS e MORRIS (1985), com o aumento do nível de lisina na ração, assim como constatado neste trabalho.

TESSERAUD et al. (1996) constataram que a deficiência em um único aminoácido afetou significativamente $(\mathrm{P}<0,01)$, o ganho de peso dos animais, por outro lado, ACAR et al. (1991), nas mesmas condições, não observaram mudanças no ganho de peso, mas sim no rendimento de cortes especiais.

Rendimento de carcaça, perna, peito, filé de peito e gordura abdominal

As médias dos resultados de peso da carcaça, peso de perna, peso de peito, peso de filé de peito e gordura abdominal referentes as aves abatidas aos 42 dias de idade, alimentadas com as dietas; Basal,

Tabela 3 - Efeito da fonte de lisina, em diferentes níveis, no desempenho de frangos de corte de 22 a 42 dias Table 3 - Effect of lysine source, at different levels, on broiler performance from 22 to 42 days

\begin{tabular}{|c|c|c|c|c|}
\hline $\begin{array}{l}\text { Tratamentos } \\
\text { Treatments }\end{array}$ & $\begin{array}{c}\text { Lisina }(\%) \\
\text { Lysine } \\
\text { Calculado\Analisado } \\
\text { Calculated } \backslash \text { Analyzed }\end{array}$ & $\begin{array}{l}\text { Ganho de peso }(\mathrm{g}) \\
\text { Body weight gain }\end{array}$ & $\begin{array}{c}\text { Consumo de ração }(\mathrm{g}) \\
\text { Feed intake }\end{array}$ & $\begin{array}{l}\text { Conv. alimentar } \\
\text { Feed:gain ratio }\end{array}$ \\
\hline $\begin{array}{l}\text { Dieta basal } \\
\text { Basal diet }\end{array}$ & $0,809 \backslash 0,790$ & $1221^{\mathrm{b}}$ & $2758^{b}$ & $2,260^{\mathrm{a}}$ \\
\hline $\begin{array}{l}\text { Lisina } \mathrm{HCl} 0,08 \\
\text { Lysine }\end{array}$ & $0,889 \backslash 0,880$ & 1408 & 2985 & 2,210 \\
\hline $\begin{array}{l}\text { Lisina } \mathrm{HCl} 0,16 \\
\text { Lysine }\end{array}$ & $0,969 \backslash 0,960$ & 1489 & 2980 & 2,001 \\
\hline $\begin{array}{l}\text { Lisina } \mathrm{HCl} 0,24 \\
\text { Lysine }\end{array}$ & $1,049 \backslash 1,000$ & 1474 & 2920 & 1,981 \\
\hline $\begin{array}{l}\text { Média } \\
\text { Mean }\end{array}$ & $0,969 \backslash 0,947$ & $\begin{array}{l}1457^{\mathrm{a}} \\
\mathrm{L}^{*}\end{array}$ & $\begin{array}{l}2951^{\mathrm{a}} \\
\mathrm{NS}\end{array}$ & $\begin{array}{l}2,030^{\mathrm{b}} \\
\mathrm{L} * *\end{array}$ \\
\hline $\begin{array}{l}\text { Lisina sulfato } 0,08 \\
\text { Lysine sulfate }\end{array}$ & $0,889 \backslash 0,860$ & 1402 & 2911 & 2,076 \\
\hline $\begin{array}{l}\text { Lisina sulfato } 0,16 \\
\text { Lysine sulfate }\end{array}$ & $0,969 \backslash 0,960$ & 1481 & 2989 & 2,018 \\
\hline $\begin{array}{l}\text { Lisina sulfato } 0,24 \\
\text { Lysine sulfate }\end{array}$ & $1,049 \backslash 1,070$ & 1499 & 3021 & 2,015 \\
\hline $\begin{array}{l}\text { Média } \\
\text { Mean } \\
\text { CV }(\%)\end{array}$ & $0,969 \backslash 0,963$ & $\begin{array}{l}1461^{\mathrm{a}} \\
\mathrm{L} * * \\
3,817\end{array}$ & $\begin{array}{l}2986^{\mathrm{a}} \\
\mathrm{L} * * \\
3,159\end{array}$ & $\begin{array}{l}2,047^{b} \\
\mathrm{NS} \\
3,152\end{array}$ \\
\hline
\end{tabular}

NS Não significativo (Not significant). * Efeito Linear (Linear effect) $(\mathrm{P}<0,05) .{ }^{* *}$ Efeito Linear (Linear effect) $(\mathrm{P}<0,01)$.

Médias nas mesma coluna seguidas de letras diferentes, diferem $(P<0,05)$, pelo teste de Newman-Keuls.

Means in the same column followed by different letters are different by Newman Keuls test. 
Tabela 4 - Efeito da fonte de lisina, em diferentes níveis, no desempenho de frangos de corte de 1 a 42 dias Table 4 - Effect of lysine source, at different levels, on broiler performance from 1 to 42 days

\begin{tabular}{|c|c|c|c|c|}
\hline $\begin{array}{l}\text { Tratamentos } \\
\text { Treatments }\end{array}$ & $\begin{array}{c}\text { Lisina }(\%) \\
\text { Lysine } \\
\text { Calculado } \backslash \text { Analisado } \\
\text { Calculated } \backslash \text { Analyzed }\end{array}$ & $\begin{array}{c}\text { Ganho de peso }(\mathrm{g}) \\
\text { Body weight gain }\end{array}$ & $\begin{array}{l}\text { Consumo de ração }(\mathrm{g}) \\
\text { Feed intake }\end{array}$ & $\begin{array}{l}\text { Conversão alimentar } \\
\text { Feed:gain ratio }\end{array}$ \\
\hline $\begin{array}{l}\text { Dieta basal } \\
\text { Basal diet }\end{array}$ & $0,837 \backslash 0,806$ & $1746^{\mathrm{b}}$ & $3817^{b}$ & $2,186^{\mathrm{a}}$ \\
\hline $\begin{array}{l}\text { Lisina } \mathrm{HCl} 0,08 \\
\text { Lysine }\end{array}$ & $0,917 \backslash 0,894$ & 2003 & $4096^{\mathrm{c}}$ & 2,045 \\
\hline $\begin{array}{l}\text { Lisina } \mathrm{HCl} 0,16 \\
\text { Lysine }\end{array}$ & $0,997 \backslash 0,971$ & 2109 & 4101 & 1,945 \\
\hline $\begin{array}{l}\text { Lisina } \mathrm{HCl} 0,24 \\
\text { Lysine }\end{array}$ & $1,077 \backslash 1,016$ & 2114 & 4045 & 1,913 \\
\hline $\begin{array}{l}\text { Média } \\
\text { Mean }\end{array}$ & $0,997 \backslash 0,960$ & $\begin{array}{l}2075^{\mathrm{a}} \\
\mathrm{L} * *\end{array}$ & $\begin{array}{l}4078^{\mathrm{a}} \\
\mathrm{NS}\end{array}$ & $\begin{array}{c}1,967^{b} \\
\mathrm{~L} * *\end{array}$ \\
\hline $\begin{array}{l}\text { Lisina sulfato } 0,08 \\
\text { Lysine sulfate }\end{array}$ & $0,917 \backslash 0,890$ & 2006 & 3998 & 1,995 \\
\hline $\begin{array}{l}\text { Lisina sulfato } 0,16 \\
\text { Lysine sulfate }\end{array}$ & $0,997 \backslash 0,974$ & 2132 & 4118 & 1,932 \\
\hline $\begin{array}{l}\text { Lisina sulfato } 0,24 \\
\text { Lysine sulfate }\end{array}$ & $1,077 \backslash 1,080$ & 2159 & 4158 & 1,928 \\
\hline Média & $0,997 \backslash 0,981$ & $2099^{a}$ & $4110^{\mathrm{a}}$ & $1,960^{\mathrm{b}}$ \\
\hline Mean & & $\mathrm{L} * *$ & $\mathrm{~L}^{* *}$ & NS \\
\hline CV $(\%)$ & & 3,296 & 2,688 & 2,909 \\
\hline
\end{tabular}

NS Não significativo (Not significant). * Efeito linear (Linear effect) $(P<0,05)$, ${ }^{* *}$ Efeito Linear (Linear effect) $(P<0,01)$.

Médias nas mesma coluna seguidas de letras diferentes, diferem $(P<0,05)$, pelo teste de Newman-Keuls.

Means in the same column followed by different letters are different by Newman Keuls test.

suplementada com Lisina $\mathrm{HCl}$ e suplementada com Lisina Sulfato estão presentes na Tabela 5.

As características de rendimentos de carcaças, perna, peito, filé e gordura abdominal não foram influenciados $(\mathrm{P}>0,05)$ pelas diferentes fontes de lisina, indicando que, para as características de carcaça, as diferentes fontes de lisina foram utilizadas pelas aves com a mesma eficiência.

Semelhantes respostas foram encontradas com suínos recebendo dietas suplementadas com lisina $\mathrm{HCl}$ e lisina sulfato para os parâmetros de rendimento de carcaça, carne magra e área de olho de lombo, DEGUSSA (1997).

Em relação à dieta basal foi detectada diferença estatística $(\mathrm{P}<0,05)$, quando comparada com ambas as dietas suplementadas com lisina para todas as características de rendimento de partes, com exceção da gordura abdominal, que não apresentou diferença significativa $(\mathrm{P}>0,05)$.

ACAR et al. (1991) encontraram resultados semelhantes para rendimento de peito de aves alimentadas com dietas contendo 0,75 e $1,15 \%$ de lisina. No entanto, com uma marca comercial de alto peso corporal, estes resultados não se repetiram. MORGAN JR. e
BILGILI (1990) também observaram aumento significativo no rendimento de peito e coxas com o aumento do teor de lisina da ração.

Quando analisados estatisticamente, os parâmetros de rendimento de partes, em porcentagem, com exceção da gordura abdominal, não diferiram dos resultados das análises com o peso (g) dos mesmos (Tabela 6). GRISONI et al. (1991) verificaram diminuição na porcentagem de gordura abdominal $(\mathrm{P}<0,05)$, com a suplementação de lisina $(0,70 \mathrm{a}$ $0,10 \%$, em rações de frangos de corte, não constatando, porém, diferenças na quantidade de gordura na cavidade abdominal.

Estes dados estão de acordo com os resultados encontrados neste trabalho, no qual foi encontrada diferença estatística $(\mathrm{P}<0,09)$, entre a porcentagem de gordura abdominal das aves alimentadas com a ração basal e a ração suplementada com Lisina Sulfato, não encontrando, no entanto, diferenças para o peso de gordura abdominal entre os tratamentos, Tabela 6. A ração suplementada com Lisina $\mathrm{HCl}$, para o parâmetro porcentagem de gordura abdominal, não diferiu da ração basal nem da ração suplementada com Lisina Sulfato. 
Tabela 5 - Efeito da fonte de lisina, em diferentes níveis, no rendimento de carcaça de frangos com 42 dias de idade Table 5 - Effect of lysine source, at different levels, on carcass yield of broiler at 42 days of age

\begin{tabular}{|c|c|c|c|c|c|c|}
\hline $\begin{array}{l}\text { Tratamentos } \\
\text { Treatments }\end{array}$ & $\begin{array}{c}\text { Lisina }(\%) \\
\text { Lysine } \\
\text { Calculado } \backslash \text { Analisado } \\
\text { Calculated } \backslash \text { Analyzed }\end{array}$ & $\begin{array}{c}\text { Carcaça }(\mathrm{g}) \\
\text { Carcass }\end{array}$ & $\begin{array}{l}\text { Perna }(\mathrm{g}) \\
\quad \operatorname{Legs}\end{array}$ & $\begin{array}{l}\text { Peito }(\mathrm{g}) \\
\text { Breast }\end{array}$ & $\begin{array}{c}\text { Filé }(\mathrm{g}) \\
\text { Breast fillet }\end{array}$ & $\begin{array}{c}\text { Gordura } \\
\text { Fat }(g)\end{array}$ \\
\hline $\begin{array}{l}\text { Dieta basal } \\
\text { Basal diet }\end{array}$ & $0,837 \backslash 0,806$ & $1189^{b}$ & $384^{\mathrm{b}}$ & $353^{b}$ & $244^{b}$ & $41,4^{\mathrm{a}}$ \\
\hline $\begin{array}{l}\text { Lisina } \mathrm{HCl} 0,08 \\
\text { Lysine }\end{array}$ & $0,917 \backslash 0,894$ & 1378 & 421 & 430 & 307 & 48,0 \\
\hline $\begin{array}{l}\text { Lisina } \mathrm{HCl} 0,16 \\
\text { Lysine }\end{array}$ & $0,997 \backslash 0,971$ & 1455 & 434 & 481 & 348 & 44,0 \\
\hline $\begin{array}{l}\text { Lisina } \mathrm{HCl} 0,24 \\
\text { Lysine }\end{array}$ & $1,077 \backslash 1,016$ & 1464 & 439 & 485 & 351 & 40,0 \\
\hline $\begin{array}{l}\text { Média } \\
\text { Mean }\end{array}$ & $0,997 \backslash 0,960$ & $\begin{array}{l}1432^{\mathrm{a}} \\
\mathrm{L} *\end{array}$ & $\begin{array}{l}431^{\mathrm{a}} \\
\mathrm{NS}\end{array}$ & $\begin{array}{l}465^{\mathrm{a}} \\
\mathrm{L} *\end{array}$ & $\begin{array}{l}335^{\mathrm{a}} \\
\mathrm{L} * *\end{array}$ & $\begin{array}{c}44,0^{\mathrm{a}} \\
\mathrm{L} *\end{array}$ \\
\hline $\begin{array}{l}\text { Lisina sulfato } 0,08 \\
\text { Lysine sulfate }\end{array}$ & $0,917 \backslash 0,890$ & 1420 & 438 & 444 & 300 & 47,0 \\
\hline $\begin{array}{l}\text { Lisina sulfato } 0,16 \\
\text { Lysine sulfate }\end{array}$ & $0,997 \backslash 0,974$ & 1482 & 445 & 487 & 353 & 44,0 \\
\hline $\begin{array}{l}\text { Lisina sulfato } 0,24 \\
\text { Lysine sulfate }\end{array}$ & $1,077 \backslash 1,080$ & 1477 & 444 & 497 & 365 & 43,0 \\
\hline $\begin{array}{l}\text { Média } \\
\text { Mean } \\
\text { CV }(\%)\end{array}$ & $\begin{array}{c}0,997 \backslash 0,981 \\
\text { NS }\end{array}$ & $\begin{array}{c}1460^{\mathrm{a}} \\
\mathrm{NS} \\
4,759\end{array}$ & $\begin{array}{l}442^{\mathrm{a}} \\
\mathrm{L}^{*} \\
5,149\end{array}$ & $\begin{array}{l}476^{\mathrm{b}} \\
\mathrm{L} * * \\
5,670\end{array}$ & $\begin{array}{l}244^{\mathrm{a}} \\
\mathrm{NS} \\
7,887\end{array}$ & $44,67^{\mathrm{a}}$ \\
\hline
\end{tabular}

Tabela 6 - Efeito de diferentes fontes de lisina no rendimento de partes de frangos de corte aos 42 dias de idade Table 6 - Effect of different lysine source on parts yield of broiler at 42 days of age

\begin{tabular}{|c|c|c|c|c|c|}
\hline $\begin{array}{l}\text { Fonte de lisina } \\
\text { Lysine source }\end{array}$ & $\begin{array}{c}\text { Carcaça }(\%) \\
\text { Carcass } \\
\end{array}$ & $\begin{array}{c}\text { Perna }(\%) \\
\text { Legs }\end{array}$ & $\begin{array}{c}\text { Peito }(\%) \\
\text { Breast }\end{array}$ & $\begin{array}{c}\text { Filé de peito }(\%) \\
\text { Breast fillet }\end{array}$ & $\begin{array}{c}\text { Gordura }(\%)^{* *} \\
\text { Fat } \\
\end{array}$ \\
\hline Basal & $64,56^{b}$ & $32,33^{\mathrm{a}}$ & $29,65^{b}$ & $20,46^{\mathrm{b}}$ & $3,47^{\mathrm{b}}$ \\
\hline $\begin{array}{l}\text { Basal } \\
\text { Lisina } \mathrm{HCl}^{*}\end{array}$ & $67,06^{\mathrm{a}}$ & $30,16^{b}$ & $32,36^{\mathrm{a}}$ & $23,41^{\mathrm{a}}$ & $3,06^{\mathrm{ab}}$ \\
\hline $\begin{array}{l}\text { Lysine } \\
\text { Lisina sulfato } \\
\text { Lysine sulfate * }\end{array}$ & $67,07^{\mathrm{a}}$ & $31,40^{b}$ & $32,77^{\mathrm{a}}$ & $23,40^{\mathrm{a}}$ & $3,03^{\mathrm{a}}$ \\
\hline C.V. $(\%)$ & 2,037 & 2,664 & 4,370 & 7,279 & 12,891 \\
\hline
\end{tabular}

Médias nas mesma coluna seguidas de letras diferentes são diferentes $(P<0,05)$, pelo teste de Newman - Keuls. ${ }^{* *}(P<0,09)$, pelo teste de Newman - Keuls, * Valores médios dos tratamentos

Means in the same column followed by different letters are different by Newman Keuls test $(P<0.05),{ }^{* *}(P<0.09)$, by Newman -Keuls test, ${ }^{*}$ Value mean of the treatments.

Biodisponibilidade da lisina sulfato em relação a lisina $\mathrm{HCl}$

Os efeitos das diferentes fontes de lisina sobre o desempenho e rendimentos de partes das aves, como demonstrados nas Tabelas 2, 3, 4, 5 e 6, mostraram haver efeito linear $(\mathrm{P}<0,05)$, para ambas as fontes de lisina sobre os dados de conversão alimentar de 1 a 21 dias, ganho de peso de 1 a 21 e 1 a 42 dias de idade das aves, peso de peito e peso de filé de peito. Não houve efeito significativo dos níveis de lisina para as demais características estudadas.
A equação referente ao ganho de peso de 1 a 21 dias de idade das aves $\mathrm{Y}=544,72+439,62 \mathrm{X}_{1}+475,84 \mathrm{X}_{2}$, com um $\mathrm{R}^{2}=0,90$ (Figura 1), revela que a adição de $0,01 \%$ de Lisina $\mathrm{HCl}$ e lisina sulfato proporcionam um aumento no ganho de peso de 4,40 e 4,76 gramas respectivamente. Com base nestes dados, utilizando o modelo de regressão linear múltipla e a relação dos coeficientes de regressão, a biodisponibilidade da Lisina Sulfato foi calculada em 108,24\%, em relação a Lisina $\mathrm{HCl}$ (padronizada a 100\%), com intervalo de confiança de 90,02 a 126,46\% mostrando então para esta carac- 


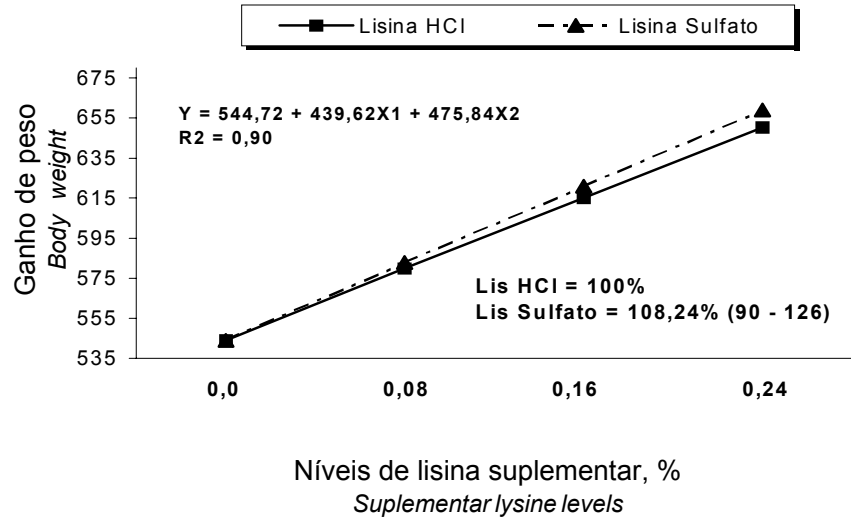

Figura 1 - Biodisponibilidade da Lisina Sulfato, em relação a Lisina $\mathrm{HCl}$, em função do ganho de peso no período de 1 a 21 dias de idade.

Figure 1 - Bioavailability of sulfate lysine and lysine $\mathrm{HCl}$ in function of weight gain of broilers from 1-21 days of age.

terística, não haver diferença significativa entre as diferentes fontes de lisina.

Para a variável ganho de peso de 1 a 42 dias de idade das aves (Figura 2), a equação estimada foi $\mathrm{Y}=1824,63+1469,18 \mathrm{X}_{1}+1381,33 \mathrm{X}_{2}$, com $\mathrm{R}^{2}=0,85$. Esta equação evidencia aumento no ganho de peso de 14,69 e 13,81 g, com a suplementação de $0,01 \%$ de Lisina $\mathrm{HCl}$ e Lisina sulfato, respectivamente. A biodisponibilidade da Lisina Sulfato, calculada em função desta característica foi de $94,02 \%$, com intervalo de confiança de 73,03 a 115,01, mostrando não haver diferença significativa entre as fontes do aminoácido estudado.

A equação referente à conversão alimentar aos 21 dias de idade das aves $\mathrm{Y}=1,9623-0,9043 \mathrm{X}_{1}-1,0235$ $\mathrm{X}_{2}$, com $\mathrm{R}^{2}=0,83$ (Figura 3 ), revela que a adição de $0,01 \%$ de Lisina $\mathrm{HCl}$ e lisina sulfato proporcionam diminuição na conversão alimentar de 0,009 e 0,010, respectivamente. Com base nestes dado, utilizando o modelo de regressão linear múltipla e a relação dos coeficientes de regressão, a biodisponibilidade da Lisina Sulfato foi calculada em 113,19\%, em relação à Lisina $\mathrm{HCl}$ (padronizada a 100\%), com intervalo de confiança de 87,46 a $138,92 \%$ mostrando, então, para esta variável não haver diferença significativa entre as diferentes fontes de lisina.

Em relação às variáveis de rendimento de carcaça, como está demostrado na Tabela 5, houve efeito linear $(\mathrm{P}<0,05)$, para ambas as fontes de lisina para as variáveis peso de peito e peso de filé de peito das aves abatidas aos 42 dias de idade. Não houve efeito

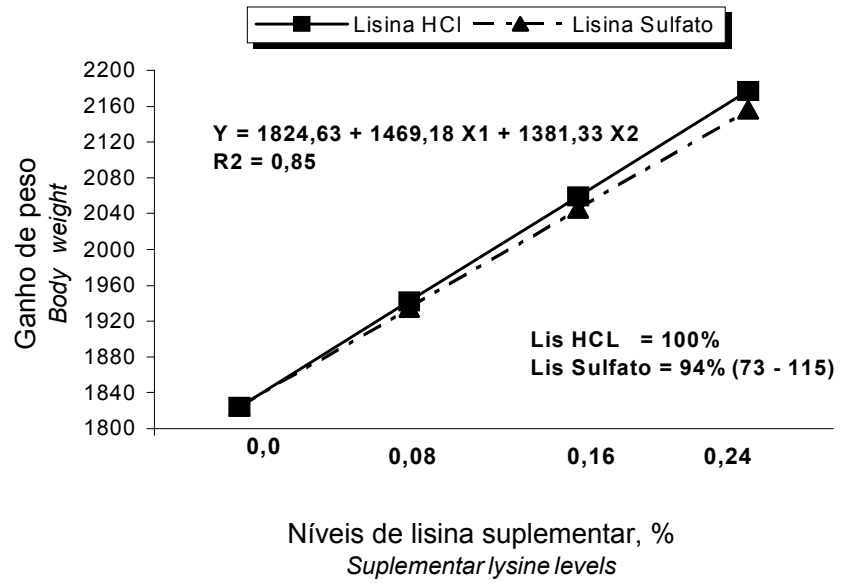

Figura 2 - Biodisponibilidade da Lisina Sulfato, em relação a Lisina $\mathrm{HCl}$, em função do ganho de peso no período de 1 a 42 dias de idade.

Figure 2 - Bioavailability of sulfate lysine and lysine $\mathrm{HCl}$ in function of weight gain of broilers from 1-42 days of age.

significativo dos níveis de lisina para as demais características estudadas.

As equações estimadas pelo modelo de regressão linear múltipla e a relação dos coeficientes de regressão para peso do peito foram; $\mathrm{Y}=0,3766+0,5320 \mathrm{X}_{1}$ $+0,4986 \mathrm{X}_{2}$, com $\mathrm{R}^{2}=0,88$ (Figura 4). Para esta característica a biodisponibilidade encontrada foi de $93,71 \%$, com intervalo de confiança de 74,88 a $112,53 \%$, não havendo, portanto, diferença significativa entre as diferentes lisinas. Os coeficientes desta

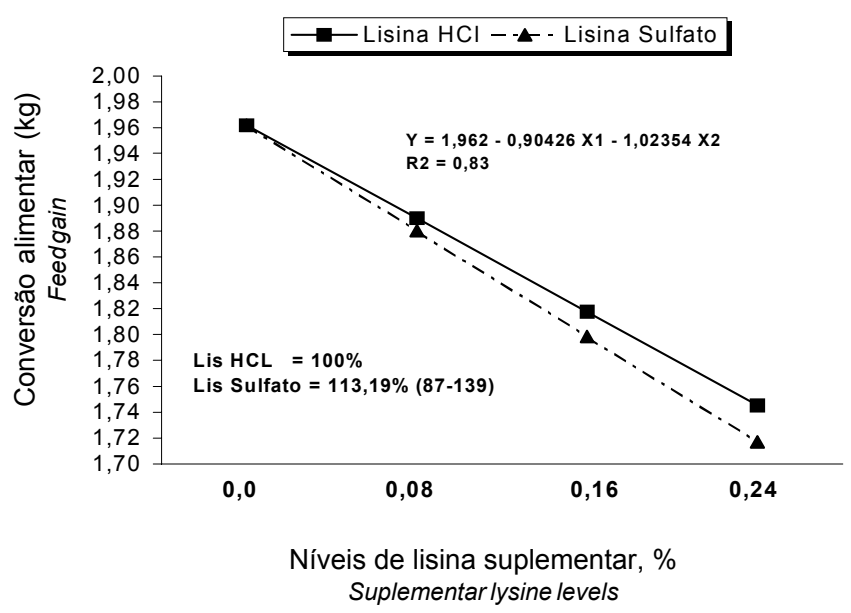

Figura 3 - Biodisponibilidade da Lisina Sulfato, em relação a Lisina $\mathrm{HCl}$ em função da Conversão Alimentar no Período de 1 a 21 dias de idade das aves.

Figure 3 - Bioavailability of sulfate lysine and lysine $\mathrm{HCl}$ in function of feed conversion of broilers from 42 days of age. 
equação mostraram acréscimo de 5,32 e 4,98 gramas de ganho em carne de peito, com a inclusão de $0,01 \%$ de Lisina $\mathrm{HCl}$ e Sulfato, respectivamente, Figura 4. A equação obtida para peso de filé de peito, $\mathrm{Y}=0,2565+0,4685 \mathrm{X}_{1}+0,4300 \mathrm{X}_{2}$, com $\mathrm{R}^{2}=0,92$, demonstra ocorrer uma deposição de carne de filé de peito de 4,68 e 4,30 g para as Lisinas $\mathrm{HCl}$ e Sulfato, respectivamente (Figura 5).

A biodisponibilidade da Lisina Sulfato, em função desta variável, foi de $91,79 \%$ com intervalo de confiança de 76,94 a 106,64, mostrando mais uma vez não haver diferença significativa entre as fontes testadas neste trabalho sob o peso do filé de peito das aves aos 42 dias de idade.

Os resultados apresentados indicam não haver diferenças $(\mathrm{P}>0,05)$, entre as duas fontes de aminoácidos, para as variáveis peso de peito, conversão alimentar aos 21 dias, ganho de peso aos 42 dias e peso de filé de peito das aves aos 42 dias de idade. As equações determinadas e utilizadas nesta pesquisa encontram-se relacionadas na Tabela 7 .

Inevitavelmente, ao se alterarem os níveis de

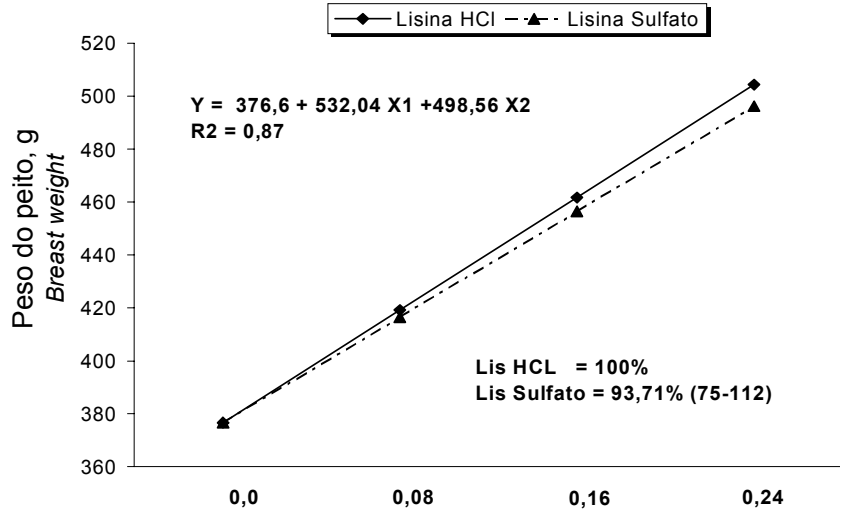

Níveis de lisina suplementar, \% Suplementarlysine levels

Figura 4 - Biodisponibilidade da Lisina Sulfato, em relação a Lisina $\mathrm{HCl}$, em função do peso do peito das aves aos 42 dias de idade.

Figure 4 - Bioavailability of sulfate lysine and lysine $\mathrm{HCl}$ in function of breast wieght of broilers at 42 days of age.

Tabela 7 - Sumário das equações de regressão linear múltiplas, nível de significância, disponibilidade média e coeficiente de correlação $\left(R^{2}\right)$

Table 7 - Summary of multiple linear regression equations, significant levels, availability mean and correlation coefficient $\left(R^{2}\right)$

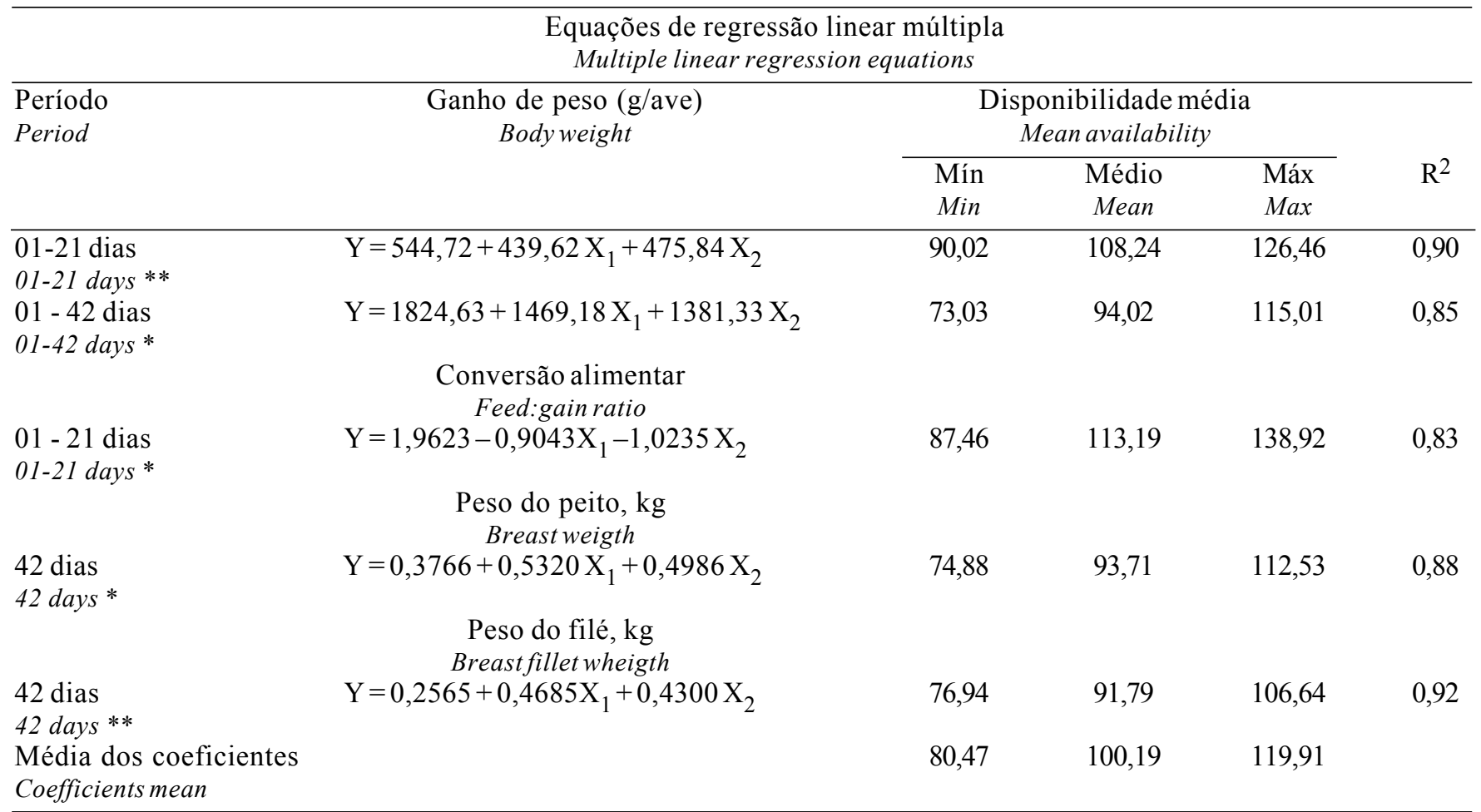

${ }^{*}(P<0,05)$ pelo teste $F,{ }^{* *}(P<0,01)$ pelo teste $F$.

${ }^{*}(P<.05)$ by $F$ test, ${ }^{* *}(P<.01)$ by test $F$. 


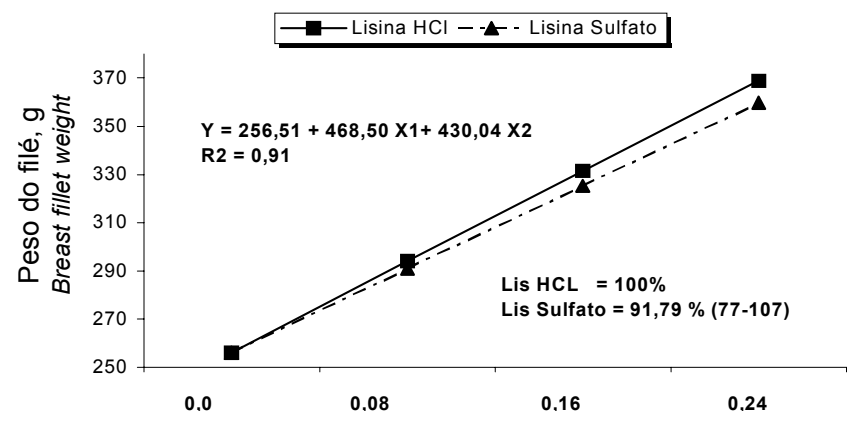

Níveis de lisina suplementar, \% Supplementarlysine levels

Figura 5 - Biodisponibilidade da Lisina Sulfato, em relação à Lisina $\mathrm{HCl}$, em função do peso do filé de peito das aves aos 42 dias de idade.

Figure 5 - Bioavailability of sulfate lysine and lysine $\mathrm{HCl}$ in function of breast fillet wieght of broilers at 42 days of age.

suplementação de lisina das rações, para testar somente seu efeito no desempenho animal, altera-se também a relação aminoacídica destas rações, criando desta maneira mais uma fonte de variação, que pode ter influenciado os resultados obtidos. Por outro lado, o intuito deste trabalho não é determinar o melhor nível de lisina nas rações para frangos de corte, nas quais está possível variação teria um peso significativo, mas sim comparar duas formas de lisina disponíveis no mercado.

\section{Conclusões}

Para os resultados de desempenho; ganho de peso, consumo de ração e conversão alimentar e os resultados de rendimento de partes; carcaça, perna, peito, filé de peito e gordura abdominal, não foram encontrados efeitos das diferentes fontes de lisina $(\mathrm{P}>0,05)$. A biodisponibilidade encontrada para a Lisina Sulfato em relação a Lisina $\mathrm{HCl}$, para as características ganho de peso de 1 a 21 e 1 a 42 dias de idade das aves, conversão alimentar (1-21 dias), peso de carne de peito e peso de filé de peito foi de $100,19 \%$, mostrando não haver diferença significativa, pela comparação dos coeficientes de regressão, entre a biodisponibilidade das lisinas testadas.

A Lisina Sulfato e a Lisina $\mathrm{HCl}$ apresentaram valores semelhantes de biodisponibilidade da lisina. As fontes de lisina estudadas poderão ser utilizadas em rações de frangos de corte, sem comprometer o desempenho e o rendimento de carcaça das aves.

\section{Referências Bibliográficas}

ACAR, N., MORAN JR., E.T., BILGILI, S.F. 1991. Live performance and carcass yield of male broilers from two commercial strain crosses receiving rations containing lysine below and above the established requirement between 6 and 8 weeks of age. Poult. Sci., 70(11):2315-2321.

ALBINO, L.F.T. Sistema de avaliação nutricional de alimentos e suas aplicações na formulação de rações para frangos de corte. Viçosa, MG: UFV, 1991. 141p. Tese (Doutorado em Zootecnia) - Universidade Federal de Viçosa, 1991.

AUSTIC, R.E. 1985. Lysine in poultry nutrition. [S.1]: Ajinomoto.

BAKER, D.H.,HAN, Y. 1994. Ideal amino acid profile for chicks during the first three weeks posthatching. Poult. Sci., 73(9):1441-1447.

BARBOSA, R.J. Exigência de metionina + cistina para frangos de corte na fase de crescimento e acabamento. Viçosa, MG:UFV, 1998. 84p. Dissertação (Mestrado em Zootecnia) - Universidade Federal de Viçosa, 1998.

DEGUSSA. 1997. An evaluation of two different lysine soucers: comparison of Biolys 60 vs. L-Lysine HCl in diets for growing finishing pigs on growth performance and carcass characteristics. Frankfurt:(Feed back facts \& figures, n.3; FF/1403/MR/04.98).

EMMERT, J.L., DOUGLAS, M.W., BOLING, E.D. et al. 1999. Bioavailability of lysine from a liquid lysine source in chicks. Poult. Sci., 78(3):383-386.

GOUS, R.M., MORRIS, T.R. 1985. Evaluation of a diet dilution technique for meansuring the response of broiler chickens to increasing concentrations oflysine. Brit. Poult. Sci., 26(2):147-161.

GRISONI, M.L., UZU, G., LARBIER, M. et al. 1991. effect of dietary lysine level on lipogenesis in broilers. Reprod. Nutr. Dev., 31:683-690.

MORAN JR., E.T, BILGILI, S.F. 1990. Processing losses, carcass quality and meat yields for broiler chicken receiving diets marginally deficient to adequate in lysine prior to marketing. Poult. Sci., 69(4):702-710.

PACK, M. Proteína ideal para frango de corte. Conceitos e posição atual. In: CONFERÊNCIA APINCO DE TECNOLOGIA AVÍCOLAS, 1995, Curitiba. Anais... Curitiba: FACTA, 1995. p.95-110.

PARSONS, C.M., BAKER, D.H. The concept and use of ideal proteins in the feeding of nonruminants. In: REUNIÃO ANUAL DA SOCIEDADE BRASILEIRA DE ZOOTECNIA, 31, 1994, Maringá. Anais... Maringá: SBZ, 1994. p.120-128.

ROSTAGNO, H.S., BARBARINO JR., P., BARBOSA, W.A. Exigências nutricionais as aves determinadas no Brasil. In: SIMPÓSIO INTERNACIONAL SOBRE EXIGÊNCIAS NUTRICIONAIS DE AVES E SUÍNOS, 1996, Viçosa, MG. Anais...Viçosa, MG: DZO, 1996. p.361-388.

SAEG. Sistema para Análise Estatística e Genética, versão 7.0. Viçosa: Fundação Arthur Bernardes, 1997.

SASSE, C.E., BAKER, D.H. 1973. Availability of sulfur amino acids in corn and corn glutem meal for growing chicks. J. Anim. Sci., 37(6):1351-1355.

TESSERAUD, S., MAAA, N., PERESSON, R. et al. 1996. Relative responses of protein turnover in three different skeletal muscles to dietary lysine deficiency in chicks. Brit. Poult. Sci., 37(3):641-650.

WALdROUP., P.W., MITSHELL, R.J., PAYNE, J.R. 1976. Performance of chicks fed diets formulated to minimize excess levels of essential amino acids. Poult. Sci., 55(1):243-253. 